\title{
miR-96 promotes breast cancer metastasis by suppressing MTSS1
}

\author{
WEI XIE ${ }^{1 *}$, FENG SUN $^{2 *}$, LIN CHEN $^{3}$ and XINJIAN CAO ${ }^{1}$ \\ ${ }^{1}$ Department of Laboratory Medicine, Nantong First People's Hospital, The Second Affiliated Hospital of Nantong University, \\ Nantong, Jiangsu 226001; ${ }^{2}$ Department of Laboratory Medicine, Nantong Tumor Hospital, Nantong, Jiangsu 226361; \\ ${ }^{3}$ Department of Laboratory Medicine, Nantong Third People's Hospital, Nantong, Jiangsu 226000, P.R. China
}

Received September 24, 2016; Accepted October 13, 2017

DOI: $10.3892 / 01.2018 .7728$

\begin{abstract}
Novel, non-invasive biomarkers with high sensitivity and specificity are critical for breast cancer treatment, and prognosis. MicroRNA (miR)-96 has been demonstrated to be highly expressed in several solid malignancies, including breast cancer. However, its expression and function in the metastasis and prognosis of breast cancer have not been fully explored, and its regulation mechanisms remain unclear. In the present study, the serum miR-96 expression in healthy controls, benign and malignant breast cancer types was compared by using reverse transcription-quantitative polymerase chain reaction. The effect of chemotherapy on miR-96 expression in breast cancer was also investigated. Result revealed that miR-96 expression was increased in malignant breast cancer types and reduced in patients following chemotherapy treatment. The effect of miR-96 manipulation on the migration of breast cancer cells was also investigated by using wound healing, and Transwell migration assays. These results revealed that the induced expression of miR96 led to enhanced wound closing and trans-membrane cell numbers. By using bioinformatics analysis, western blotting and immunohistochemical staining, the metastasis suppressor-1 (MTSS1) gene was identified to be the functional target of miR-96 in the promotion of cell migration. In conclusion, it was identified that miR-96 exhibited an increased level in serum samples of patients with malignant breast cancer in comparison with benign breast tumor types and health controls and may be substantially reduced by chemotherapy treatment, implying that it may be used as a prognostic marker in breast cancer. miR-96 overexpression may inhibit migration of breast cancer cells by downregulating MTSS1 expression.
\end{abstract}

Correspondence to: Professor Xinjian Cao, Department of Laboratory Medicine, Nantong First People's Hospital, The Second Affiliated Hospital of Nantong University, 6 North Haier Lane Road, Nantong, Jiangsu 226001, P.R. China

E-mail: nantongujs@163.com

${ }^{*}$ Contributed equally

Key words: microRNA-96, breast cancer, metastasis, metastasis suppressor-1

\section{Introduction}

Breast cancer, a highly heterogeneous disease, is one of the most commonly diagnosed types of cancer and the second most common cause of cancer-associated mortality in women globally $(1,2)$. Breast cancer metastasizes to the distant organs and an increased number of patients with breast cancer with earlier stages survive their disease for at least 5 years compared with patients diagnosed with cancer metastasis (3). Detection of breast cancer at an early stage is important to improve breast cancer prognosis and reduce the mortality of this disease (4). At present, mammography and ultrasound have been successfully used in the screening of early-stage breast cancer (5). However, novel non-invasive biomarkers are required to optimize individual treatment.

Small non-coding microRNAs (miRNAs/miRs) are epigenetic regulators that mediate specific cellular mRNA degradation processes and inhibit translation to modulate gene expression post-transcriptionally (6). Dysregulation of miRNA expression is involved in the initiation and progression, including metastasis, proliferation, chemoresistance, and recurrence of breast, prostate, lung and colon cancer (5-8). Increasing evidence has indicated that miRNAs may serve as tumor oncogenes or anti-oncogenes of types of human cancer, including breast cancer (9-11). Several studies have demonstrated that serum and plasma miRNAs (circulating miRNAs) present potential as novel non-invasive biomarkers for the early diagnosis of various types of cancer (12-14). In breast cancer, studies have suggested that the various breast cancer subtypes exhibit different molecular miRNA signatures $(14,15)$. miRNAs have been identified to be stable in whole blood, plasma, serum, saliva and urine, and they have been proposed as potentially accessible breast cancer biomarkers for clinical use $(10,14,16,17)$. miRNA expression profiling of breast cancer has identified signatures associated with diagnosis, staging, progression, prognosis and response to treatment $(10,14,15)$. For example, several miRNA-based signatures have been identified with notably high predictive values including a 3-miRNA (miR-199a, miR-29c, and miR-424) signature with an area under the curve (AUC) of 0.888 and a 7-miRNA panel with an AUC of 0.914 in sera samples of patients with breast cancer $(18,19)$.

miR-96 belongs to the same family as miR-183, and is a well-recognized oncogenic miRNA in a variety of types of cancer, including hepatocellular carcinoma, prostate cancer, 
medulloblastoma, pancreatic cancer, colorectal carcinoma and breast cancer (20-24). A previous study demonstrated that the upregulation of miRNA-96 targeting Forkhead box protein (FOX)O3a served a notable function in the pro-proliferation effect of breast cancer and hepatoma cells $(25,26)$. In addition, miR-96 is overexpressed in papillary thyroid carcinoma and prostate cancer cells, and functions as an oncogene through repressing FOXO1 expression $(27,28)$. In breast cancer, the overexpression of miR-96 was also demonstrated to induce the migration of breast cancer cells by downregulating transcriptional factors FOXO3a, and FOXO1 (29). However, the expression of serum miR-96 in the metastasis and prognosis of breast cancer has not been fully explored and its regulation mechanisms remain unclear. Metastasis suppressor-1 (MTSS1) is a metastasis suppressor gene which was first identified in non-metastatic bladder cancer cell lines (30). Functional and mechanism analysis suggested that MTSS1 protein may be associated with cancer progression or tumor metastasis in a variety of organ sites $(31,32)$. Whether miR-96 targets MTSS1 dysregulation function in breast cancer metastasis remains unknown.

In the present study, the expression of serum miR-96 was confirmed in healthy control, benign and malignant breast cancer samples. Then, the effect of miR-96 expression on chemotherapy prognosis was examined. The migration ability of induced miR-96 overexpression in breast cancer cells was also analyzed. Finally, the mechanism of direct targeting of MTSS1 by miR-96 in breast cancer was explored. These results identified the function and mechanism of miR-96 in the breast cancer progression, and prognosis. Serum miR-96 may be used as a novel therapeutic and prognostic marker in breast cancer.

\section{Materials and methods}

Cell culture and miR-96 transfection. The human breast cancer MCF-7 and MDA-MB-231 cell lines were obtained from the Shanghai Institute of Biochemistry and Cell Biology of the Shanghai Institutes of Biological Sciences, Chinese Academy of Sciences (Shanghai, China). The cell lines were maintained in plastic flasks as adherent monolayers in high glucose Eagle's minimal essential medium (H-DMEM) supplemented with $10 \%$ fetal bovine serum (FBS; Invitrogen; Thermo Fisher Scientific, Inc., Waltham, MA, USA) and incubated at $37^{\circ} \mathrm{C}$ in a humidified atmosphere supplemented with $5 \% \mathrm{CO}_{2}$.

The cells were transfected with an miR-96 mimics and inhibitors by using Lipofectamine ${ }^{\mathrm{TM}} 2000$ (Invitrogen; Thermo Fisher Scientific, Inc.). In order to induce overexpression of miR-96, MCF-7 and MDA-MB-231 were treated with different concentrations of miR-96 mimics and inhibitors (12.5, 25 and 50 nM) (Shanghai GenePharma Biotechnology, Co., Ltd., Shanghai, China). The sequences of the miR-96 mimics were designed as follows: 5'-UUUGGCACUAGCACAUUUUUG CU-3' (sense) and 5'-CAAAAAUGUGCUAGUGCCAAA UU-3' (antisense). The sequence of the miR-96 inhibitors was designed as follows: 5'-AGCAAAAAUGUGCUAGUGCCA AA-3'. The cells were harvested $48 \mathrm{~h}$ post-transfection, and total RNA was extracted for reverse transcription-quantitative polymerase chain reaction (RT-qPCR) analysis. Cell lysates were prepared for western blot analysis.
Clinical tissue samples. Breast cancer tissues and adjacent control were collected from 5 female patients (aged from 36 to 65 years, with an average age 52.6 years) with breast cancer from the Nantong Tumor Hospital (Nantong, China) who had undergone total or partial mastectomy surgery between March 2015 and May 2016. The breast cancer tissues and adjacent non-cancerous tissues were histologically confirmed. All clinical procedures followed the protocols approved by the Ethical Committee of Nantong Tumor Hospital, and the methods were performed in accordance with the approved guidelines. Written informed consent was obtained from all patients prior to sample collection.

RNA extraction and miRNA RT-qPCR assay. Blood samples were collected from 118 female patients (aged from 18 to 76 years, with an average age 56 years) with breast cancer from the Nantong Tumor Hospital (Nantong, China) who had undergone total or partial mastectomy surgery between December 2014 and May 2016. Informed written consent was provided by all the patients. Each sample was centrifuged to collect sera at $1,500 \times \mathrm{g}, 4^{\circ} \mathrm{C}$ for $10 \mathrm{~min}$ and stored at $-70^{\circ} \mathrm{C}$ for RNA extraction. miRNA was isolated from $500 \mu \mathrm{l}$ serum using miRNAeasy kit (Qiagen, Inc., Valencia, CA, USA) according to the manufacturer's protocol. RNA concentration was determined by NanoDrop ND1000 spectrophotometer (NanoDrop Technologies; Thermo Fisher Scientific, Inc., Pittsburgh, PA, USA). Following treatment with DNase (Life Technologies), the RNA was eluted with $50 \mu 1$ RNAse-free water.

Serum miR-96 expression was quantified using the miScript SYBR Green PCR Kit (Qiagen GmbH, Hilden, Germany) and an ABI Prism 7900HT Real Time PCR System. The thermocycling conditions were as follows: Preheating at $95^{\circ} \mathrm{C}$ (for $5 \mathrm{~min}$ ), followed by 40 cycles at $95^{\circ} \mathrm{C}$ (for $30 \mathrm{sec}$ ) and $60^{\circ} \mathrm{C}$ (for $45 \mathrm{sec}$ ). The primer for miR-96 was forward, 5'-GCCCGC TTTGGCACTAGCACATT-3' and reverse, 5'-GTGCAGGGT CCGAGGT-3'. U6 small nuclear RNA was used as an internal control and the primer was forward, 5'-TGCGGGTGCTCG CTTCGGCAGC-3' and reverse, 5'-CCAGTGCAGGGTCCG AGGT-3'. All the serum samples were analyzed in triplicate. The relative expression of the miR-96 was calculated using the comparative cycle threshold $\left(2^{-\Delta \Delta \mathrm{Cq}}\right)$ method and normalized to U6 (33).

Western blot analysis. Cultured cells were harvested and lysed in RIPA buffer [10 mM Tris (pH 7.4), $150 \mathrm{mM} \mathrm{NaCl}$, $1 \mathrm{mM}$ EGTA, $0.1 \% \mathrm{SDS}$ ] supplemented with proteinase inhibitors ( $1 \mathrm{mM} \mathrm{NaF}, 1 \mathrm{mM} \mathrm{Na} 3 \mathrm{VO} 4,1 \mathrm{mM}$ PMSF, $1 \mathrm{mg} / \mathrm{ml}$ aprotinin and $1 \mathrm{mg} / \mathrm{ml}$ leupeptin; cat no. E211-02; Vazyme, Piscataway, NJ, USA). Protein concentration was determined using an BCA assay. Equal quantities of protein $(20 \mu \mathrm{g})$ were loaded and separated with $10 \%$ SDS-PAGE, transferred to methanol pre-activated polyvinylidene fluoride membranes and blocked in $5 \%(\mathrm{w} / \mathrm{v})$ non-fat milk at room temperature for $1 \mathrm{~h}$. Western blot analysis was performed to detect the expression of various proteins using the following primary antibodies: MTSS1 (1:1,000; cat no. SC-101204; Santa Cruz Biotechnology Inc., Dallas, TX, USA), E-cadherin (1:2,000; cat no. SC-7870; Santa Cruz Biotechnology Inc.), N-cadherin (1:1,500; cat no. SC-1502; Santa Cruz Biotechnology Inc.), 
vimentin (1:2,000; cat no. BS-1491; Biogot Technology Co., Ltd., Nanjing, China) and GAPDH (1:3,000; cat no. KC-5G4; Zhejiang Kangchen Biotech Co., Ltd., Wuhan, China). Following incubation with the primary antibodies overnight at $4^{\circ} \mathrm{C}$, membranes were washed 3 times with tris-buffered saline containing $0.05 \%$ Tween-20 and incubated with horseradish peroxidase-conjugated anti-rabbit (1:5,000; cat no. CW0103S; CWBIO Biotech Inc., Beijing, China) or anti-mouse (1:5,000; cat no. CW0102S; CWBIO Biotech Inc.) secondary antibodies at room temperature for $1 \mathrm{~h}$. Proteins were detected with an ECL enhanced chemiluminescence detection system (GE Healthcare, Chicago, IL, USA).

Wound healing assay. MDA-MB-231, and MCF-7 cells $\left(2 \times 10^{5}\right)$ were seeded in six-well plates and cultured in H-DMEM with $10 \% \mathrm{FBS}$ at $37^{\circ} \mathrm{C}$ to reach $95 \%$ confluence. A wound, $0.35 \mathrm{~mm}$ in width, was generated by scraping with a $10 \mu$ l pipette tip. Images of the cells in the wounded monolayer were captured at 24,48 and $72 \mathrm{~h}$, and cell migration was assessed by measuring the gap sizes at five fields under a light microscope (Ti; Nikon Corporation, Tokyo, Japan) at magnification of x100.

Transwell migration assay. A Transwell migration assay was performed using a specialized chamber pre-coated with a thin layer of basement membrane matrix (ECMatrix) (Merck KGaA, Darmstadt, Germany). Medium containing 10\% FBS was placed in the lower chambers to act as a chemoattractant. Cells $\left(5 \times 10^{5}\right)$ in a $300 \mu \mathrm{l}$ serum-free medium were placed in the upper chambers and incubated at $37^{\circ} \mathrm{C}$ for 24,48 and $72 \mathrm{~h}$. Invasive cells on the lower surface of the membrane, which had migrated through the polycarbonate membrane, were stained with $10 \%$ hematoxylin at room temperature for 2 min and counted under a light microscope (Ti; Nikon Corporation) in five selected fields at magnification of x200.

Immunohistochemistry. Breast cancer tissue slides were fixed in $4 \%$ formaldehyde solution at room temperature overnight. These slides were incubated with MTSS1, E-cad, N-cad and vimentin antibodies overnight at $4{ }^{\circ} \mathrm{C}$ according to the manufacturer's protocol. The signals were visualized with 3,3'-diminobenzidine (Wuhan Boster Biological Technology, Ltd, Wuhan, China) and counterstained with $10 \%$ hematoxylin at room temperature for $2 \mathrm{~min}$. The morphological sections were evaluated and imaged with high-power light microscopy (Nikon Corporation, Tokyo, Japan) at magnification of x200.

Prediction of miR-96 target. Targets of miR-96 were predicted using online TargetScan software with a search term of has-mir-96 (Release 3.1: October 2016, URL http://www.targetscan.org/mamm_31/).

Statistical analysis. Statistical analysis was performed using SPSS software (version 22.0; IBM Corp., Armonk, NY, USA). Data are expressed as the mean \pm standard deviation. Differences of miR-96 expression in serum samples and cancer cells between two groups were analyzed using a Student's t-test (two-tailed). Differences among three groups were compared using one-way analysis of variance with Bonferroni post-hoc test. $\mathrm{P}<0.05$ was considered to indicate a statistically significant difference.

\section{Results}

Dysregulated serum miR-96 in patients with breast cancer. To verify the role of miR-96 in breast cancer, sera samples were collected from patients with breast cancer $(n=44)$, benign breast tumors $(n=18)$ and health controls $(n=18)$. RT-qPCR assays were used to detect the level of miR-96 in different groups. The results indicated that the level of miR-96 was significantly elevated in breast cancer samples compared with benign breast tumors and health controls ( $\mathrm{P}<0.05$; Fig. 1A). Subsequently, miR-96 expression was compared between the patients with breast cancer with $(n=26)$ or without chemotherapy $(n=18)$. Notably, higher expression of miR-96 was detected in the blood samples from the patients with breast cancer without chemotherapy $(\mathrm{P}<0.05$; Fig. 1B) compared with the patients who has undergone chemotherapy. The data indicate that miR-96 may be used as biomarker for breast cancer diagnosis and therapeutic outcomes.

To additionally explore the association between the expression levels of miR-96 and prognosis of patients with breast cancer, the miR-96 expression in different breast cancers was compared based on their clinical features, surface markers and clinical stages. As indicated in Fig. 1C and D, the expression of miR-96 was almost equivalent, even slightly increased in cases of estrogen receptor (ER)+ and progesterone receptor (PR)+ breast cancer compared with cases of ER- and PR- cancer. No associations between the miR-96 level and the human epidermal growth factor 2 (HER2)/neu receptor ( $P>0.05$; Fig. 1E) or between the levels of miR-96 and stages of breast cancer $(\mathrm{P}>0.05$; Fig. $1 \mathrm{~F})$ were observed.

Effects of miR-96 on breast cancer cell migration. Epithelial-to-mesenchymal transition (EMT) is a critical process in the progression of breast cancer. The present study investigated the effects of miR-96 manipulation on EMT-associated proteins expression in breast cancer cell lines. Downregulation of epithelial markers and upregulation of mesenchymal markers serves an important role in EMT. Synthetic mimics and inhibitors were employed to increase and suppress endogenous miR-96 expression in MDA-MB-231, and MCF-7 cells ( $\mathrm{P}<0.001$; Fig. $2 \mathrm{~A}$ and $\mathrm{B})$. It was demonstrated that miR-96 overexpression in MDA-MB-231 and MCF-7 cell lines resulted in the downregulation of epithelial marker E-cad and the upregulation of the mesenchymal markers $\mathrm{N}$-cad and vimentin (Fig. 2C). It was additionally identified that miR-96 knockdown promoted E-cad expression and inhibited $\mathrm{N}$-cad expression. Vimentin downregulation was observed in MDA-MB-231 cells, but not in MCF-7 cells (Fig. 2D). These data reinforce that MDA-MB-231 and MCF-7 may represent different types of breast cancer.

Next, the biological effects of miR-96 in migration of breast cancer cells was investigated. The results of the wound healing assay indicated that the migration of MDA-MB-231 and MCF-7 cells were enhanced by $25 \mathrm{nM}$ miR-96 mimics ( $\mathrm{P}<0.05$; Fig. 3A) at $48 \mathrm{~h}$ post-treatment. Consistently, miR-96 inhibitors treatment suppressed the migration of MDA-MB-231 and MCF-7 cells at $72 \mathrm{~h}(\mathrm{P}<0.05$; Fig. 3B). The Transwell migration assay also revealed that miR-96 upregulation significantly promoted the migration of breast cancer cells $(\mathrm{P}<0.01$; Fig. 4A). miR-96 suppression also decreased the migration of breast cancer cells 

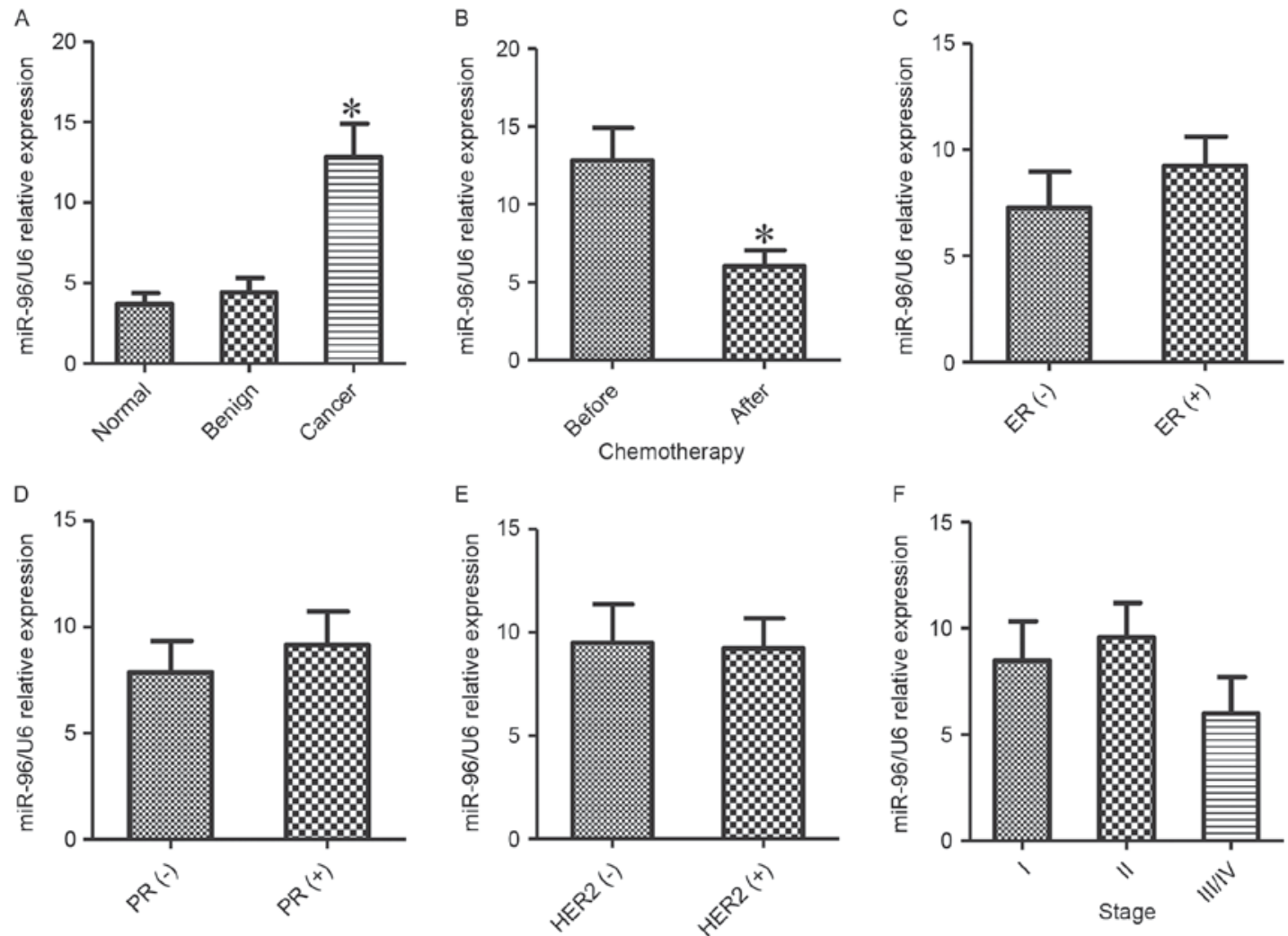

Figure 1. Serum miR-96 was highly expressed in patients with breast cancer and associated with breast cancer prognosis. (A) Serum miR-96 levels from patients with breast cancer $(\mathrm{n}=44)$, benign breast tumors $(\mathrm{n}=18)$ and health controls $(\mathrm{n}=18)$ were detected with RT-qPCR. ${ }^{*} \mathrm{P}<0.05$ vs. adjacent benign breast tumors (B) miR-96 levels in patients with breast cancer prior and subsequent to chemotherapy were detected with RT-qPCR. "P<0.05 vs. breast patients prior to chemotherapy. Serum miR-96 levels in patients with (C) estrogen receptor (ER)+ and ER-, (D) progesterone receptor (PR)+ and PR- and (E) HER2+ and HER2-breast cancer were detected with RT-qPCR. (F) Serum miR-96 levels in patients with clinical stages I, II, III and IV were detected with RT-qPCR. RT-qPCR, reverse transcription quantitative polymerase chain reaction; miR, microRNA; ER, estrogen receptor; PR, progesterone receptor; HER2, human epidermal growth factor 2 .
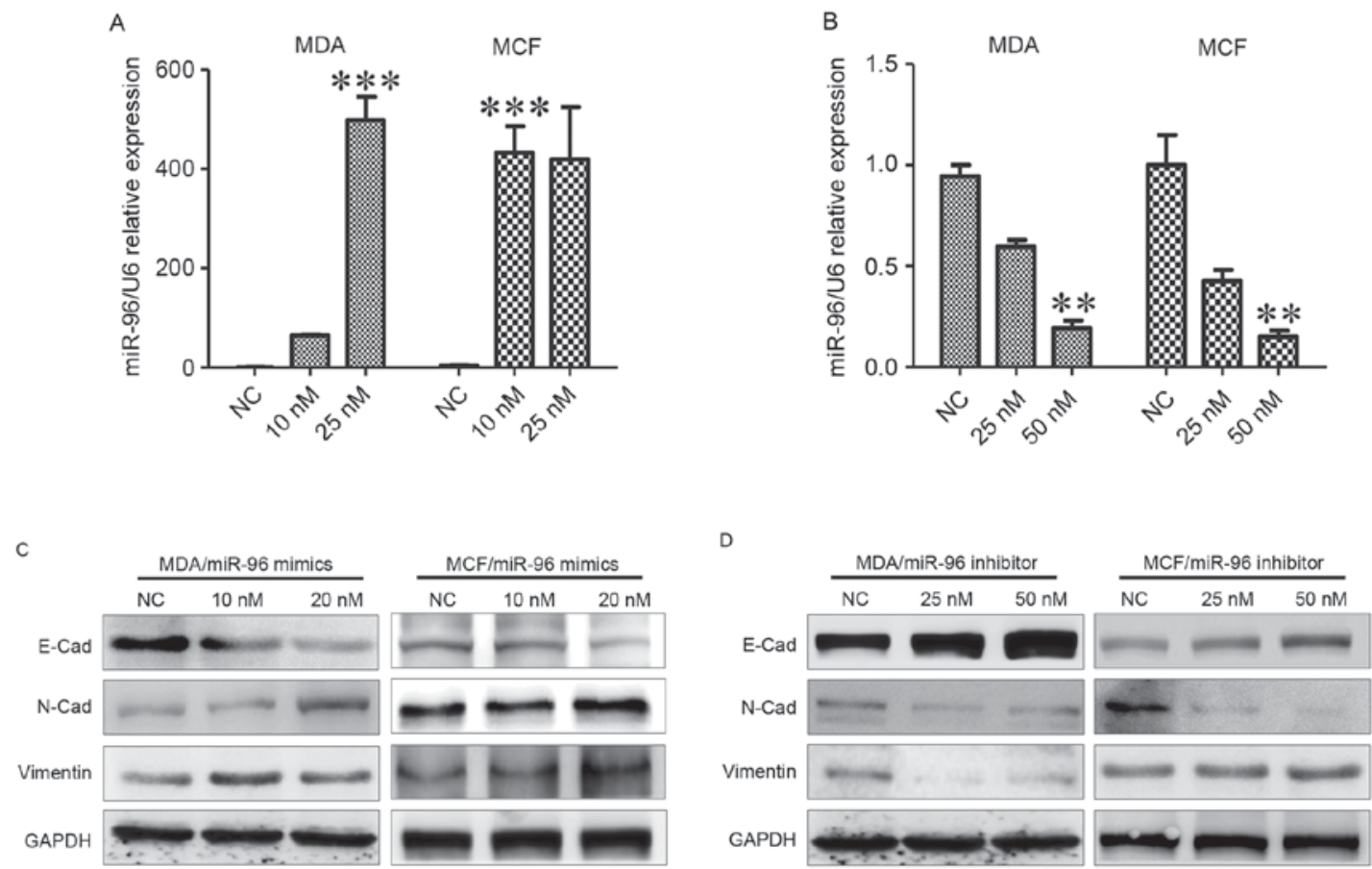

Figure 2. miR-96 promoted the expression of epithelial-to-mesenchymal transition associated proteins. (A) miR-96 was overexpressed in miR-96 mimic-treated MDA-MB-231 and MCF-7 breast cancer cell lines. ${ }^{* * *} \mathrm{P}<0.001$ vs. NC. (B) miR-96 was inhibited in miR-96 inhibitor-treated MDA-MB- 231 and MCF-7 breast cancer cell lines. ${ }^{* *} \mathrm{P}<0.01$ vs. NC. (C and D) Expression of epithelial marker (E-cad) and mesenchymal markers (N-cad and vimentin) in miR-96 mimics and inhibitor-treated MDA-MB-231 and MCF-7 cell lines were determined by western blot analysis. NC, negative control; E-cad, epithelial cadherin; N-cad, neural cadherin; miR, microRNA. 
A
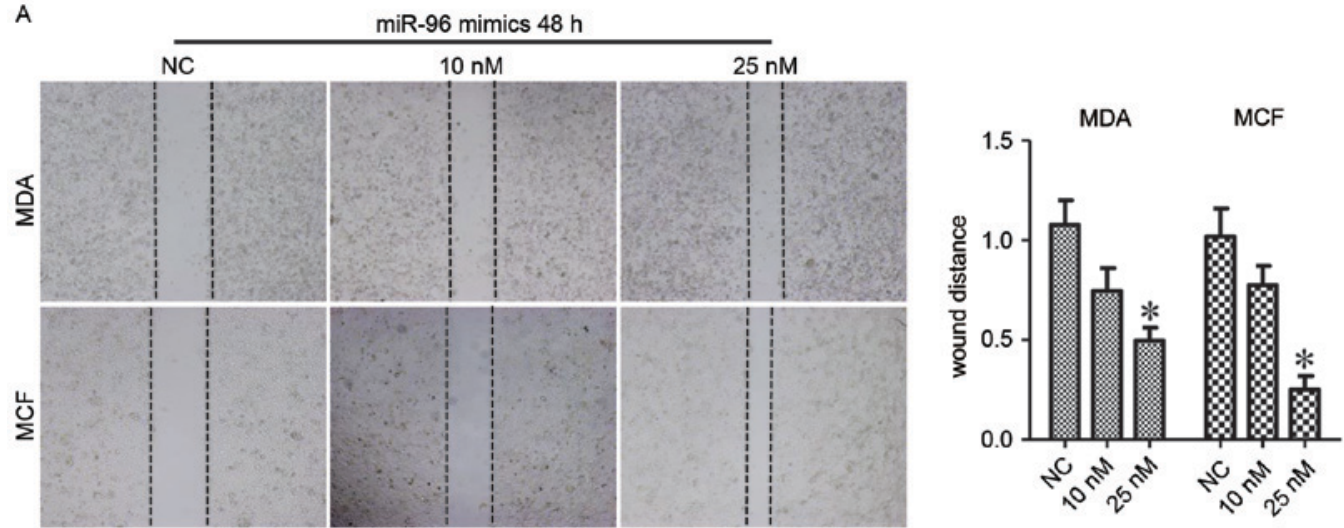

B

miR-96 inhibitor $72 \mathrm{~h}$
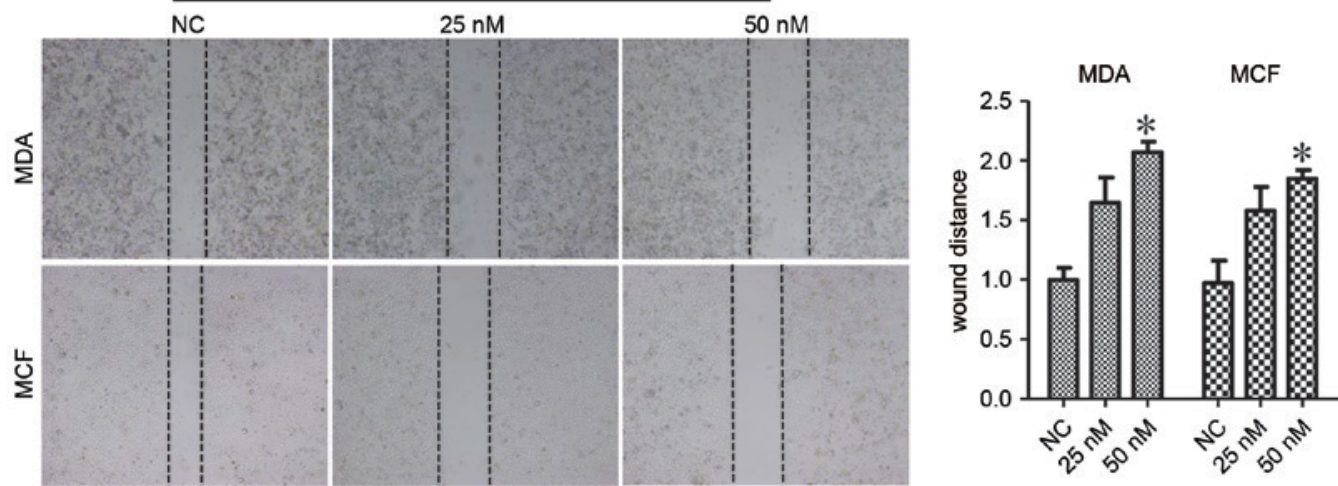

Figure 3. miR-96 promoted the migration of breast cancer cells by wound healing assay. Wound healing of MDA-MB-231 and MCF-7 cells was (A) promoted by miR-96 mimics treatment and (B) inhibited by miR-96 inhibitors treatment. "P<0.05 vs. NC. NC, negative control; miR, microRNA.
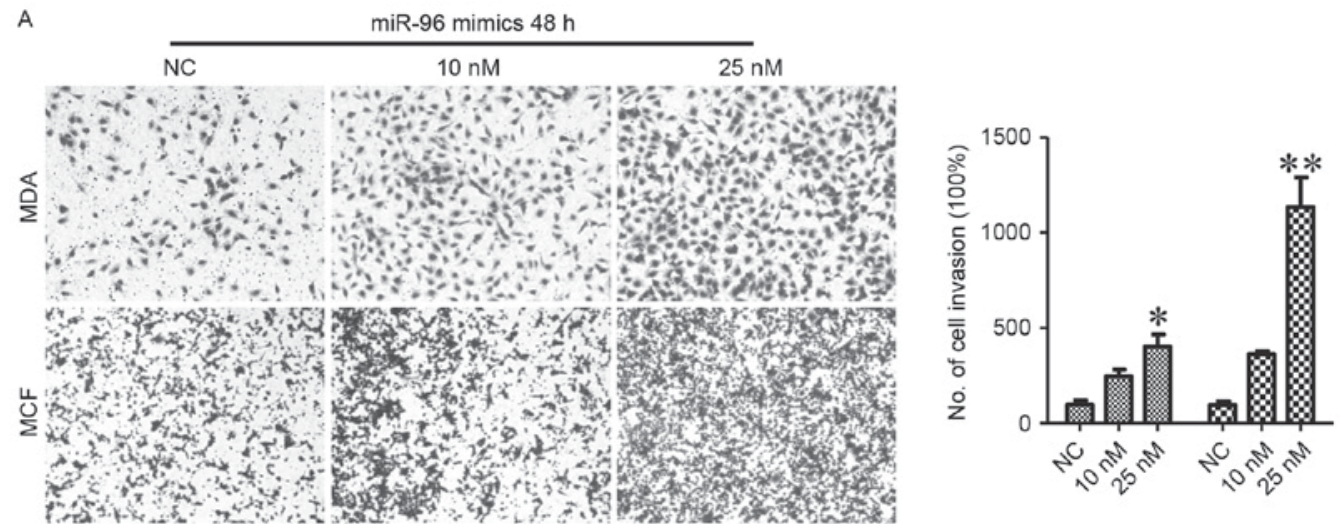

B
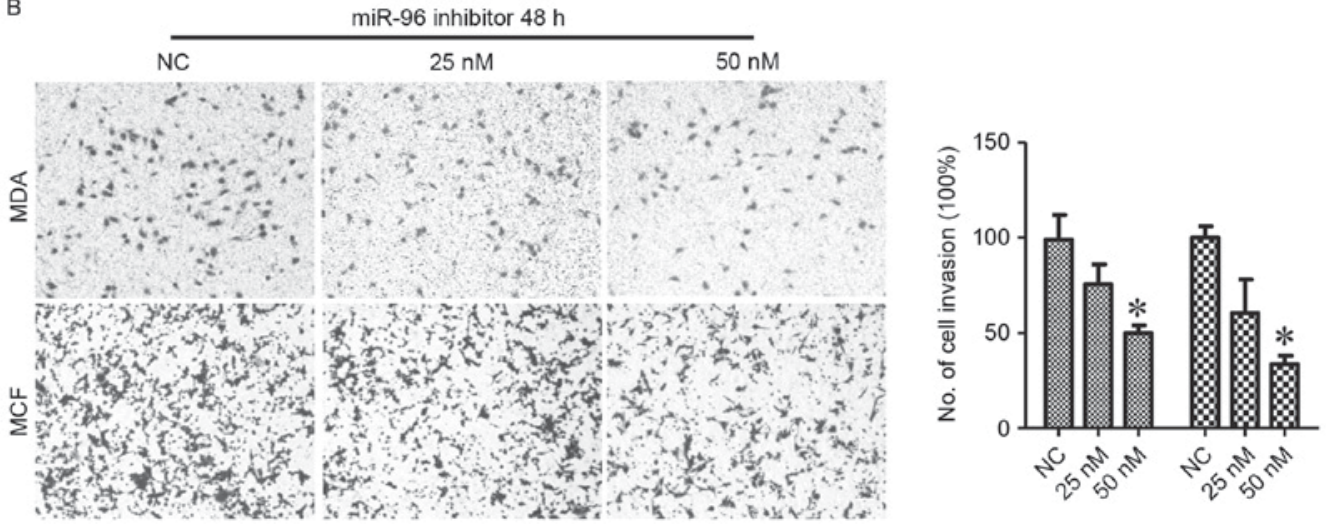

Figure 4. miR-96 promoted the migration of breast cancer cells by the Transwell migration assay. Transwell cell migration of MDA-MB-231 and MCF-7 cells was promoted by (A) miR-96 mimic treatment and inhibited by (B) miR-96 inhibitor treatment. " $\mathrm{P}<0.05$ and ${ }^{* *} \mathrm{P}<0.01$ vs. NC. NC, negative control. miR, microRNA. 
A

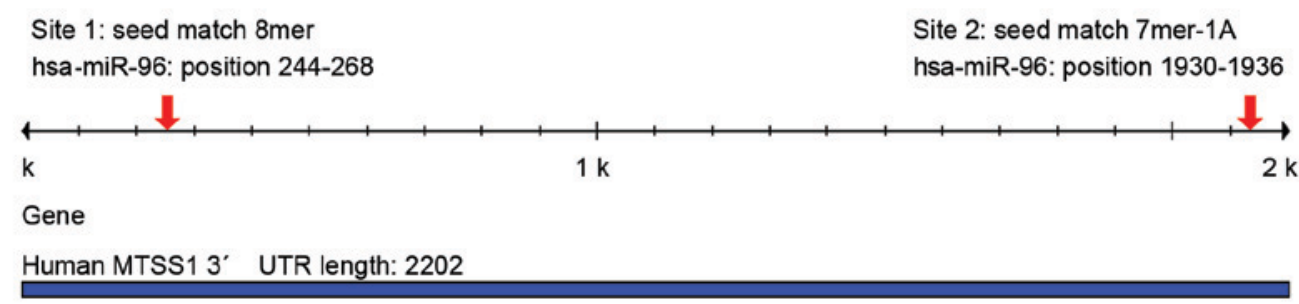

B

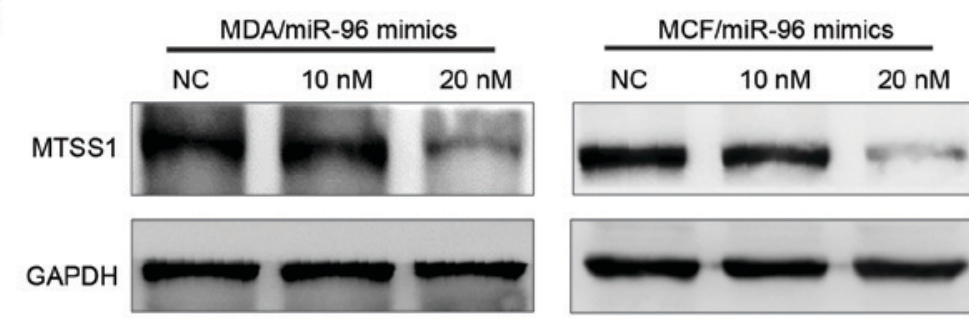

C
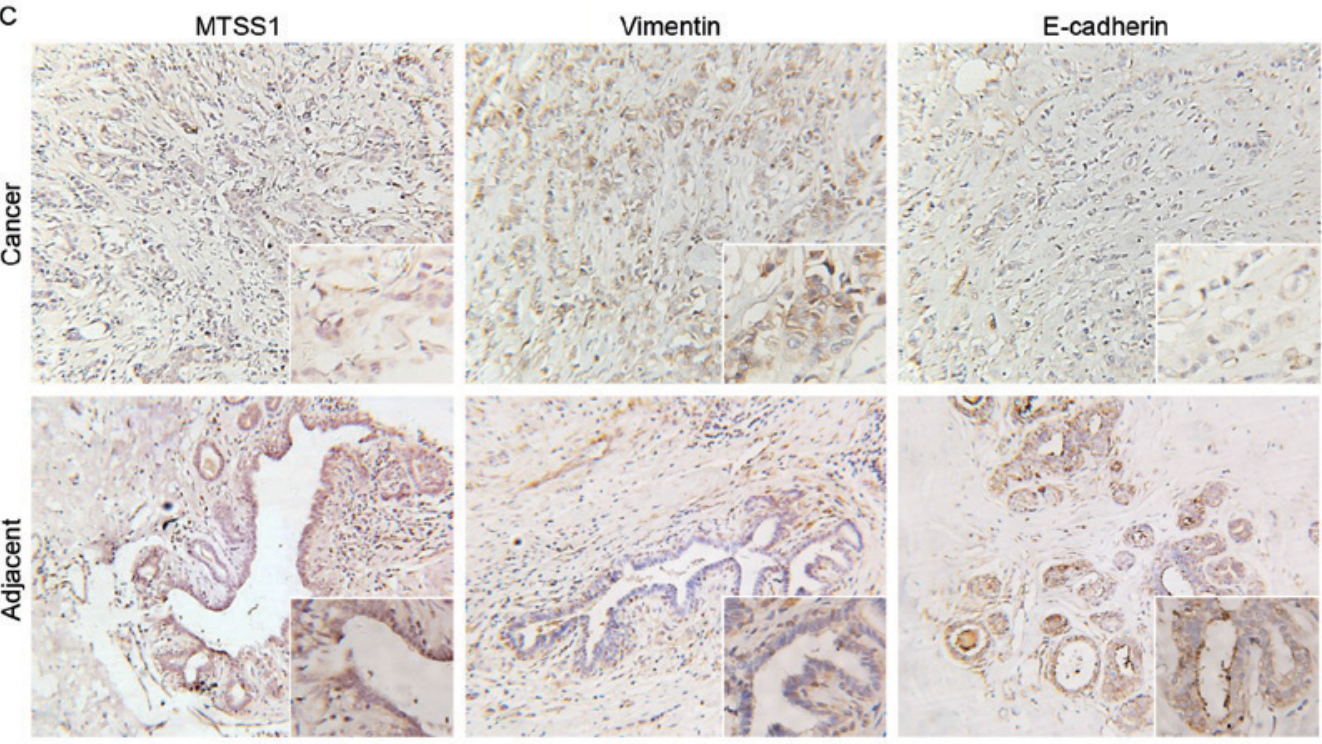

Figure 5. MTSS1 was downregulated by miR-96 in breast cancer cells. (A) The 3'UTR region of MTSS1 mRNA is partially complementary to miR-96. (B) MTSS1 expression of miR-96 mimic-treated MDA-MB-231 and MCF-7 cells was detected via western blot analysis. (C) MTSS1, vimentin and E-cadherin expression in breast cancer and adjacent noncancerous tissues was detected by immunohistochemistry (at a magnification of x200). miR, microRNA; MTSS1, metastasis suppressor 1; UTR, untranslated region; E-cadherin, epithelial cadherin.

( $\mathrm{P}<0.05$; Fig. 4B). These data indicated that miR-96 serves an important role in the maintenance of malignant phenotypes of breast cancer cells.

miR-96 suppresses MTTS1 expression in breast cancer cells. To verify the factors mediating the effect of miR-96 on breast cancer, the target of miR-96 were predicted by using online database TargetScan (Release 3.1: October 2016). A total of 2 conserved miR-96 binding sites were identified in the 3'untranslated region of the human MTSS1 gene (Fig. 5A). To additionally confirm whether the MTSS1 gene was a target of miR-96, MTSS1 expression was investigated in breast cancer tissues and miR-96-manipulated cell lines. The results of the western blot analysis indicated that treatment with miR-96 mimics suppressed MTSS1 expression and miR-96 inhibitors increased MTSS1 expression in breast cancer cells (Fig. 5B). The results of immunohistochemistry also revealed that MTSS1 was downregulated in breast cancer samples compared with noncancerous tissues (Fig. 5C). In contrast, E-cad expression was upregulated in noncancerous tissues and reduced in breast cancer samples (Fig. 5C).

\section{Discussion}

miR-96 is highly expressed in various types of cancers, including breast cancer, hepatocellular carcinoma, prostate carcinoma and non-small cell lung cancer (20-24). The role of miR-96 in the prognosis of breast cancer remains unknown. In the present study, it was identified that serum miR-96 levels were significantly increased in breast cancer cells compared with the benign and healthy controls, and miR-96 expression decreased markedly in patients with breast cancer undergoing chemotherapy. The expression of miR-96 was almost equivalent in ER+, PR+ and HER+ types of cancer compared with in ER- and PR- and HER- types of cancer. miR-96 expression was also not changed between different stages of breast cancer. 
Previously, Li et al demonstrated that miR-96 was decreased in ER+ and PR+ breast cancer and increased in HER2-enriched breast cancer (34). In the present study, 44 breast cancer samples were collected to investigate miR-96 expression in different types of breast cancer. Additional samples of breast cancer should be examined to comprehensively elucidate miR-96 expression in breast cancer.

Previous studies have demonstrated that miR-96 may increase cancer cell proliferation and migration in prostate cancer and breast cancer $(24,34)$. The data from the present study support a proto-oncogenic miRNA role for miR-96 in breast cancer cell lines, as overexpression of miR-96 by mimics in MCF-7 and MDA-MB-231 cell lines induced cell migration. The migration results of MCF-7 were similar to those demonstrated by Li et al (34), who also identified that upregulation of miR-96 promoted migration of the breast cancer MCF-7 and T47D cell lines. The present study indicated that downregulation of miR-96 by inhibitors in MCF-7 and MDA-MB-231 cell lines also decreased cell migration. $\mathrm{Xu}$ et al (24) also suggested that the invasiveness of prostate cancer cells was partially suppressed by miR-96 inhibitor treatment. Furthermore, the data of the present study revealed that the expression of epithelial marker E-cad was decreased, and mesenchymal markers $\mathrm{N}$-cad and vimentin were induced in miR-96-overexpressed breast cancer cells.

MTSS1 is known to be a metastasis suppressor, and to suppress proliferation and EMT in non-small cell lung cancer, hepatitis B-associated hepatocellular carcinoma and bladder urothelial carcinoma cells, prostate carcinoma cells, chronic myeloid leukemia and the tongue squamous cellular carcinoma Tca8113 cell line $(24,31,32,35)$. Loss of MTSS1 facilitates the progression of prostate and breast cancers. Similar to other types of cancer, MTSS1 has also been suggested to demonstrate prognostic value and anti-metastatic effects in breast cancer (36-40). The immunohistochemistry results of the present study indicated that invasive breast cancer tumors exhibited decreased expression of MTSS1 compared with paracancerous tissue, which additionally confirmed that MTSS1 is a tumor suppressor in breast cancer.

The regulation of MTSS1 is also of interest for the study of prostate cancer biology. Downregulation of MTSS1 expression contributes to the growth, development, and metastasis of breast and prostate cancer (36-38). Zhong et al (38) demonstrated that Skp, Cullin, F-box containing complex $\beta$-transducin repeat-containing protein, a E3 ubiquitin ligase complex with a function in different types of cancer including breast or prostate cancer cells, inhibited MTSS1 expression in a ubiquitination-dependent fashion. miR-15 and miR-182-5p were also identified to participate in the regulation of MTSS1 transcription in prostate cancer cells $(37,41)$. However, it is necessary to investigate the mechanisms involved with MTSS1 deregulation in breast cancer. The results of the present study revealed that miR-96 may downregulate MTSS expression in breast cancer cells.

In conclusion, miR-96 was indicated to be associated with the prognosis of patients with breast cancer, and may suppress migration and invasiveness of breast cancer cells by downregulating MTSS1 expression. The present study implied that miR-96 may be a useful therapeutic target and prognostic marker for breast cancer treatment.

\section{Acknowledgements}

The present study was supported by the Project of Nantong Science and Technology bureau guiding science and technology (grant no. HS149134).

\section{References}

1. Huang X, Li X, Xie X, Ye F, Chen B, Song C, Tang H and Xie X: High expressions of LDHA and AMPK as prognostic biomarkers for breast cancer. Breast 30: 39-46, 2016.

2. Torre LA, Bray F, Siegel RL, Ferlay J, Lortet-Tieulent J and Jemal A: Global cancer statistics, 2012. CA Cancer J Clin 65: 87-108, 2015.

3. Kakushadze Z, Raghubanshi R and Yu W: Estimating Cost Savings from Early Cancer Diagnosis. SSRN Electronic J, 2017.

4. Schootman M, Fuortes L and Aft R: Prognosis of metachronous contralateral breast cancer according to stage at diagnosis: The importance of early detection. Breast Cancer Res Treat 99: 91-95, 2006.

5. Bucchi L, Belli P, Benelli E, Bernardi D, Brancato B, Calabrese M, Carbonaro LA, Caumo F, Cavallo-Marincola B, Clauser P, et al: Recommendations for breast imaging follow-up of women with a previous history of breast cancer: Position paper from the Italian Group for Mammography Screening (GISMa) and the Italian College of Breast Radiologists (ICBR) by SIRM. Radiol Med 121: 891-896, 2016.

6. Svoronos A, Engelman D and Slack F: OncomiR or Tumor Suppressor? The Duplicity of MicroRNAs in Cancer. Cancer Res 76: 3666-3670, 2016.

7. Alhasan AH, Scott AW, Wu JJ, Feng G, Meeks JJ, Thaxton CS and Mirkin CA: Circulating microRNA signature for the diagnosis of very high-risk prostate cancer. Proc Natl Acad Sci USA 113: 10655-10660, 2016.

8. Kang J, Kim W, Lee S, Kwon D, Chun J, Son B, Kim E, Lee JM, Youn $\mathrm{H}$ and Youn B: TFAP2C promotes lung tumorigenesis and aggressiveness through miR-183- and miR-33a-mediated cell cycle regulation. Oncogene 36: 1585-1596, 2016.

9. Pichler M, Stiegelbauer V, Vychytilova-Faltejskova P, Ivan C, Ling H, Winter E, Zhang X, Goblirsch M, Wulf-Goldenberg A, Ohtsuka M, et al: Genome-wide microRNA analysis identifies miR-188-3p as novel prognostic marker and molecular factor involved in colorectal carcinogenesis. Clin Cancer Res 23: 1323-1333, 2016.

10. Hannafon BN, Trigoso YD, Calloway CL, Zhao YD, Lum DH, Welm AL, Zhao ZJ, Blick KE, Dooley WC and Ding WQ: Plasma exosome microRNAs are indicative of breast cancer. Breast Cancer Res 18: 90, 2016.

11. Liu M, Yang R, Urrehman U, Ye C, Yan X, Cui S, Hong Y, Gu Y, Liu Y, Zhao C, et al: miR-19b suppresses PTPRG to promote breast tumorigenesis. Oncotarget 7: 64100-64108, 2016.

12. Li C, Li J, Cai Q, Qiu QQ, Yan M, Liu BY and Zhu ZG: miRNA-199a-3p: A potential circulating diagnostic biomarker for early gastric cancer. J Surg Oncol 108: 89-92, 2013.

13. Qi Y, Cui L, Ge Y, Shi Z, Zhao K, Guo X, Yang D, Yu H, Cui L, Shan Y, et al: Altered serum microRNAs as biomarkers for the early diagnosis of pulmonary tuberculosis infection. BMC Infect Dis 12: 384, 2012.

14. Adhami M, Haghdoost AA, Sadeghi B and Malekpour Afshar R: Candidate miRNAs in human breast cancer biomarkers: A systematic review. Breast Cancer: Nov 3, 2017 (Epub ahead of print).

15. Ohzawa H, Miki A, Teratani T, Shiba S, Sakuma Y, Nishimura W, Noda Y, Fukushima N, Fujii H, Hozumi Y, et al: Usefulness of miRNA profiles for predicting pathological responses to neoadjuvant chemotherapy in patients with human epidermal growth factor receptor 2-positive breast cancer. Oncol Lett 13: 1731-1740, 2017.

16. Hemmatzadeh M, Mohammadi H, Jadidi-Niaragh F, Asghari $\mathrm{F}$ and Yousefi M: The role of oncomirs in the pathogenesis and treatment of breast cancer. Biomed Pharmacother 78: 129-139, 2016.

17. Mar-Aguilar F, Rodríguez-Padilla C and Reséndez-Pérez D: Use of serum-circulating miRNA profiling for the identification of breast cancer biomarkers. Methods Mol Biol 1165: 71-80, 2014. 
18. Zhang L, Xu Y, Jin X, Wang Z, Wu Y, Zhao D, Chen G, Li D, Wang $\mathrm{X}$, Cao $\mathrm{H}$, et al: A circulating miRNA signature as a diagnostic biomarker for non-invasive early detection of breast cancer. Breast Cancer Res Treat 154: 423-434, 2015.

19. Huo D, Clayton WM, Yoshimatsu TF, Chen J and Olopade OI: Identification of a circulating MicroRNA signature to distinguish recurrence in breast cancer patients. Oncotarget 7: 55231-55248, 2016.

20. Cheung CC, Lun SW, Chung GT, Chow C, Lo C, Choy KW and Lo KW: MicroRNA-183 suppresses cancer stem-like cell properties in EBV-associated nasopharyngeal carcinoma. BMC Cancer 16: 495, 2016.

21. Gokhale A, Kunder R, Goel A, Sarin R, Moiyadi A, Shenoy A, Mamidipally C, Noronha S, Kannan S and Shirsat NV: Distinctive microRNA signature of medulloblastomas associated with the WNT signaling pathway. J Cancer Res Ther 6: 521-529, 2010.

22. Leung WK, He M, Chan AW, Law PT and Wong N: Wnt/ $\beta$-Catenin activates miR-183/96/182 expression in hepatocellular carcinoma that promotes cell invasion. Cancer Lett 362 97-105, 2015.

23. Rapti S, Kontos CK, Papadopoulos IN and Scorilas A: High miR-96 levels in colorectal adenocarcinoma predict poor prognosis, particularly in patients without distant metastasis at the time of initial diagnosis. Tumour Biol 37: 11815-11824, 2016

24. Xu L, Zhong J, Guo B, Zhu Q, Liang H, Wen N, Yun W and Zhang L: miR-96 promotes the growth of prostate carcinoma cells by suppressing MTSS1. Tumour Biol 37: 12023-12032, 2016.

25. Lin H, Dai T, Xiong H, Zhao X, Chen X, Yu C, Li J, Wang X and Song L: Unregulated miR-96 induces cell proliferation in human breast cancer by downregulating transcriptional factor FOXO3a PLoS One 5: e15797, 2010

26. Xu D, He X, Chang Y, Xu C, Jiang X, Sun S and Lin J: Inhibition of miR-96 expression reduces cell proliferation and clonogenicity of HepG2 hepatoma cells. Oncol Rep 29: 653-661, 2013.

27. Haflidadottir BS, Larne O, Martin M, Persson M, Edsjö A, Bjartell A and Ceder Y: Upregulation of miR-96 enhances cellular proliferation of prostate cancer cells through FOXO1. PLoS One 8: e72400, 2013

28. Song HM, Luo Y, Li DF, Wei CK, Hua KY, Song JL, Xu H, Maskey N and Fang L: MicroRNA-96 plays an oncogenic role by targeting FOXO1 and regulating AKT/FOXO1/Bim pathway in papillary thyroid carcinoma cells. Int J Clin Exp Pathol 8: 9889-9900, 2015.

29. Gao F and Wang W: MicroRNA-96 promotes the proliferation of colorectal cancer cells and targets tumor protein p53 inducible nuclear protein 1, forkhead box protein O1 (FOXO1) and FOXO3a. Mol Med Rep 11: 1200-1206, 2015

30. Lee YG, Macoska JA, Korenchuk S and Pienta KJ: MIM, a potential metastasis suppressor gene in bladder cancer. Neoplasia 4: 291-294, 2002
31. Huang XY, Huang ZL, Xu B, Chen Z, Re TJ, Zheng Q, Tang ZY and Huang XY: Elevated MTSS1 expression associated with metastasis and poor prognosis of residual hepatitis B-related hepatocellular carcinoma. J Exp Clin Cancer Res 35: 85, 2016.

32. Li XD, Zhang JX, Jiang LJ, Wang FW, Liu LL, Liao YJ, Jin XH, Chen WH, Chen X, Guo SJ, et al: Overexpression of maelstrom promotes bladder urothelial carcinoma cell aggressiveness by epigenetically downregulating MTSS1 through DNMT3B. Oncogene 35: 6281-6292, 2016.

33. Livak KJ and Schmittgen TD: Analysis of relative gene expression data using real-time quantitative PCR and the 2(-Delta Delta C(T)) method. Methods 25: 402-408, 2001

34. Li P, Sheng C, Huang L, Zhang H, Huang L, Cheng Z and Zhu Q: miR-183/-96/-182 cluster is up-regulated in most breast cancers and increases cell proliferation and migration. Breast Cancer Res 16: 473, 2014.

35. Guo Y, Ren M, Shang C, Zhu L and Zhong M: MTSS1 gene regulated by miR-96 inhibits cell proliferation and metastasis in tongue squamous cellular carcinoma Tca8113 cell line. Int J Clin Exp Med 8: 15441-15449, 2015.

36. Giacobbe A, Compagnone M, Bongiorno-Borbone L, Antonov A, Markert EK, Zhou JH, Annicchiarico-Petruzzelli M, Melino G and Peschiaroli A: p63 controls cell migration and invasion by transcriptional regulation of MTSS1. Oncogene 35: 1602-1608, 2016.

37. Kedmi M, Ben-Chetrit N, Körner C, Mancini M, Ben-Moshe NB, Lauriola M, Lavi S, Biagioni F, Carvalho S, Cohen-Dvashi H, et al: EGF induces microRNAs that target suppressors of cell migration: miR-15b targets MTSS1 in breast cancer. Sci Signal 8: ra29, 2015.

38. Zhong J, Shaik S, Wan L, Tron AE, Wang Z, Sun L, Inuzuka H and Wei W: SCF $\beta$-TRCP targets MTSS1 for ubiquitination-mediated destruction to regulate cancer cell proliferation and migration. Oncotarget 4: 2339-2353, 2013.

39. Hicks DG, Yoder BJ, Short S, Tarr S, Prescott N, Crowe JP, Dawson AE, Budd GT, Sizemore S, Cicek M, et al: Loss of breast cancer metastasis suppressor 1 protein expression predicts reduced disease-free survival in subsets of breast cancer patients. Clin Cancer Res 12: 6702-6708, 2006.

40. Parr C and Jiang WG: Metastasis suppressor 1 (MTSS1) demonstrates prognostic value and anti-metastatic properties in breast cancer. Eur J Cancer 45: 1673-1683, 2009.

41. Hirata H, Ueno K, Shahryari V, Deng G, Tanaka Y, Tabatabai ZL, Hinoda Y and Dahiya R: MicroRNA-182-5p promotes cell invasion and proliferation by down regulating FOXF2, RECK and MTSS1 genes in human prostate cancer. PLoS One 8: e55502, 2013.

This work is licensed under a Creative Commons Attribution-NonCommercial-NoDerivatives 4.0 International (CC BY-NC-ND 4.0) License. 